\title{
Modelo matemático estocástico para diagnosticar calidad de agua y sedimentos en zonas influenciadas por la actividad petrolera en la provincia de Orellana.
}

Stochastic mathematical model to diagnose water and sediment quality in areas influenced by oil activity in the province of Orellana.

\author{
Carlos Rolando Rosero Erazo. ${ }^{1}$, Fausto Manolo Yaulema Garces. ${ }^{2} \&$ Jorge Luis \\ Yaulema Castañeda. ${ }^{3}$
}

\begin{abstract}
DOI: https://doi.org/10.33262/concienciadigital.v4i3.1789

Introduction. Contamination of water by heavy metals due to oil activity constitutes one of the problems of greatest concern at the local, regional and global levels, since it constitutes a danger to aquatic biota and human health. Objective. Determine the stochastic mathematical model to diagnose water and sediment quality in areas influenced by oil activity in the province of Orellana, Methodology. Data was collected through the 24 monitoring points currently active within the province, in the case of sediments, except Mini Culebra station, because they do not have data for the established years 2015-2019. The concentrations of the non-conservative compounds under study were recorded, such

1 Escuela Superior Politécnica de Chimborazo, Facultad de Ciencias. Riobamba, Ecuador carlos.roseroe@espoch.edu.ec, https://orcid.org/0000-0003-2691-5578

2 Escuela Superior Politécnica de Chimborazo, Facultad de Recursos Naturales. Riobamba, Ecuador. fyaulema@espoch.edu.ec, https://orcid.org/0000-0002-5793-976X

3 Escuela Superior Politécnica de Chimborazo, Facultad de Ciencias. Riobamba, Ecuador jorge.yaulema@espoch.edu.ec, https://orcid.org/0000-0002-0646-3984
\end{abstract}


as: cadmium, nickel, lead and total petroleum hydrocarbons, through descriptive statistics, the river or natural channel of the first order (Napo river) and the second order (river Payamino, Coca River, Jivino River, Huamayacu River, Blanco River and Sacha River). Results. Once the mathematical model was applied in the SWAT modeling, it indicated that the TPH cover a greater range of sedimentation with a concentration of $18,704.4$ to $90080 \mathrm{mg} /$ ha in most micro-watersheds, the sedimentation of (Ni) ranges from 1332.68 to $1512.34 \mathrm{mg} / \mathrm{ha}$. With the highest concentration in the micro-basins of the north and central-east zone, the concentration of $(\mathrm{Pb})$ ranges from 269,948 to 323.55 $\mathrm{mg} / \mathrm{ha}$ in the micro-basins of the north zone, and lastly $(\mathrm{Cd})$ which is the pollutant with the lowest sedimentation presents with a concentration of 49.49 to $187.88 \mathrm{mg} / \mathrm{ha}$, Conclusion. The data obtained exceed the carrying capacity, assuming a virtual accumulation of said pollutants analyzed in the rivers, so it is recommended to establish an environmental management plan for the control and treatment of hydrocarbon emissions caused by oil activity in the province of Orellana.

Keywords: Soil pollution, Heavy metals, Water quality, Hydrocarbons

\section{Resumen.}

Introducción. La contaminación del agua por metales pesados debido a la actividad petrolera constituye uno de los problemas de mayor preocupación a nivel local, regional y mundial, debido a que constituye un peligro para la biota acuática y la salud humana. Objetivo. Determinar el modelo matemático estocástico para diagnosticar calidad de agua y sedimentos en zonas influenciadas por la actividad petrolera en la provincia de Orellana, Metodología. Se realizó la recolección de datos a través de los 24 puntos de monitoreo actualmente activos dentro de la provincia, en el caso de sedimentos, excepto Mini estación Culebra, debido a que no poseen datos de los años establecidos 2015-2019. Se registró las concentraciones de los compuestos no conservativos en estudio como son: cadmio, níquel, plomo e hidrocarburos totales de petróleo, mediante una estadística descriptiva, se determinó el río o canal natural de primer orden (río Napo) y de segundo orden (río Payamino, río Coca, río Jivino, río Huamayacu, río Blanco y río Sacha). Resultados. Una vez aplicado el modelo matemático en el modelamiento SWAT indicó que los TPH cubren un mayor rango de sedimentación con una concentración de 18704.4 a $90080 \mathrm{mg} / \mathrm{ha}$ en la mayoría de microcuencas, la sedimentación de (Ni) va de 1332.68 a $1512.34 \mathrm{mg} / \mathrm{ha}$ con mayor concentración en las microcuencas de la zona norte y centroeste, la concentración de $(\mathrm{Pb})$ va de 269.948 a $323.55 \mathrm{mg} / \mathrm{ha}$ en las microcuencas de la zona norte, y por ultimo $(\mathrm{Cd})$ que es el contaminante que menor sedimentación presenta con una concentración de 49.49 a $187.88 \mathrm{mg} / \mathrm{ha}$, Conclusión. los datos obtenidos exceden la capacidad de carga, suponiendo una acumulación virtual de dichos contaminantes analizados en los ríos, por lo que se recomienda establecer un plan de manejo ambiental para el control y tratamiento de las emisiones hidrocarburíferas ocasionadas por la actividad petrolera en la provincia de Orellana.

Palabras clave: Contaminación del suelo, Metales pesados, Calidad del agua, Hidrocarburos 


\section{Introducción}

A lo largo de los años el Ambiente a nivel global ha sufrido pérdida y disminución de su calidad ambiental, consecuencia de las actividades humanas como la explotación de recursos naturales y la presión socioambiental a la que se ha sometido provocando el deterioro de los ecosistemas y la perdida de renovabilidad de sus recursos. La provincia de Orellana no es una excepción ya que es considerada como un hotspot de biodiversidad producto de su situación geográfica y de unas condiciones climatológicas especiales. Estas características también han favorecido una alta presencia de recursos naturales como hidrocarburos, madera, agricultura.

La explotación de hidrocarburos han sido una de las principales atracciones económicas históricas de la provincia si bien esto ha provocado también una alta presencia de contaminación derivada de dicha actividad y en especial en el sector rural, donde también se acumula la mayor presión de pobreza. Si bien es necesario monitorear la calidad del Ambiente, las zonas con alta densidad de extracción de recursos naturales y actividades hidrocarburíferas con el fin de prevenir a tiempo y mitigar de forma rápida posibles daños ambientales.

\section{Metodologia.}

\section{Unidad de Análisis}

La provincia de Orellana ubicada al nororiente del país cuenta con una superficie de 21 730,05『km】^2 y una población de 150977 habitantes, se caracteriza por ser una de las provincias con más actividad petrolera del país, esta se encuentra dividida den 4 cantones y 33 parroquias (28 parroquias rurales y 5 urbanas) (Gobierno Autónomo Descentralizado de la Provincia de Orellana, 2019)

\section{Recolección de datos}

Los datos de las concentraciones de agua y sedimentos de la provincia de Orellana se los obtuvo gracias a la colaboración del GADPO, a través de estudios anteriores cuyos resultados reposan en los archivos departamentales de la institución. Dichos datos se obtuvieron de los 24 puntos existentes de monitoreo activos ubicados dentro de la provincia excepto el punto Mini estación Culebra el cual no posee datos de los años establecidos (2015-2019).

Tabla 1 Ríos seleccionados antes y después de la descarga

\begin{tabular}{|c|c|c|c|}
\hline Ríos & Descripción & Caudal promedio & $Q=\left(m^{3} / s\right)$ \\
\hline Payamino & Antes & & 40.34 \\
\hline Napo & Después & & 42.50 \\
\hline Ríos & Descripción & \multicolumn{2}{|c|}{ Caudal promedio $Q=\left(\mathrm{m}^{3} / \mathrm{s}\right)$} \\
\hline Coca & Antes & & 19.86 \\
\hline Napo & Después & & 17.50 \\
\hline
\end{tabular}




\begin{tabular}{lrr}
\hline Ríos & Descripción & Caudal promedio $Q=\left(\mathrm{m}^{3} / \mathrm{s}\right)$ \\
Jivino & Antes & 7.51 \\
Napo & Después & 17.50 \\
Ríos & Descripción & \\
Huamayacu & Antes & Caudal promedio $Q=\left(\mathrm{m}^{3} / \mathrm{s}\right)$ \\
Napo & Después & 5.84 \\
Ríos & Descripción & 12.50 \\
Blanco & Antes & Caudal promedio $Q=\left(\mathrm{m}^{3} / \mathrm{s}\right)$ \\
Napo & Después & 5.01 \\
Ríos & Descripción & \\
Sacha & Antes & Caudal promedio $Q=\left(\mathrm{m}^{3} / \mathrm{s}\right)$ \\
7.51 \\
\hline
\end{tabular}

Tabla 2 Coordenadas de los puntos de monitoreo

\begin{tabular}{|c|c|c|c|c|c|}
\hline Puntos de monitoreo & $\mathbf{X}$ & $\mathbf{Y}$ & $\begin{array}{l}\text { Puntos de } \\
\text { monitoreo }\end{array}$ & $\mathbf{X}$ & $\mathbf{Y}$ \\
\hline $\begin{array}{c}\text { Palo Azul - La Unión la } \\
\text { Moreña }\end{array}$ & 280819.0 & 9979701.0 & Auca Central & 290011.0 & 9928856.0 \\
\hline $\begin{array}{l}\text { Sacha Norte } 2 \text { - } \\
\text { Enokanqui }\end{array}$ & 293839.0 & 9975291.0 & Auca Sur & 290140.0 & 9921892.0 \\
\hline Sacha Norte 1 & 292989.0 & 9965884.0 & Auca 51 & 291211.0 & 9932706.0 \\
\hline Sacha Central & 291138.0 & 9961630.0 & Anaconda 1 & 294612.0 & 9942183.0 \\
\hline Sacha 192 & 296131.0 & 9971666.0 & Yuca Central & 301507.0 & 9947932.0 \\
\hline Nuevo Paraiso & 273088.0 & 9960967.0 & $\begin{array}{r}\text { Mini Estación } \\
\text { Culebra }\end{array}$ & 289487.0 & 9945960.0 \\
\hline Huashito & 272694.0 & 9955560.0 & Yulebra 1 & 292337.0 & 9946419.0 \\
\hline Estación Coca & 269905.0 & 9954387.0 & Mono 1 - CPF & 272232.0 & 9907280.0 \\
\hline Tigüino & 284072.0 & 9875996.0 & Mono 6 - SUR & 272352.0 & 9905740.0 \\
\hline Puma 2 & 282800.0 & 9923394.0 & Oso 9 & 263054.0 & 9924935.0 \\
\hline Nantu A & 303462.0 & 9916947.0 & Lobo 3 & 275401.0 & 9941727.0 \\
\hline Pindo Central & 299429.0 & 9923999.0 & Oso B & 261387.0 & 9923896.0 \\
\hline
\end{tabular}

\section{Selección del río o canal natural}

Se seleccionaron los ríos de primer orden (río Napo) y de segundo orden (río Payamino, Coca, Jivino, Huamayacu, río Blanco y río Sacha), debido a la cercanía de los puntos de monitoreo y a la existencia de información hídrica de antes y después. 


\section{Constitución de un modelo matemático}

Se considera que en los modelos de calidad es necesario la hidrología utilizando modelos matemáticos que relacionen las entradas de aguas residuales con la calidad del agua del cuerpo receptor (Osina, 2011)

Las partes constitutivas de los modelos estocásticos para la calidad del agua están compuestas por variables físicas como la temperatura y la salinidad. Variables químicas y biológicas como: oxígeno disuelto, demanda bioquímica de oxígeno, coliformes fecales, fosforo orgánico y lo que se analiza en este estudio que son los metales pesados (Arreguín, Alcocer, \& Hernández, 2010).

De la descarga de los contaminantes en la fuente receptora, y el caudal promedio es la media ponderada de los datos proporcionados por dicha información de cada uno de los ríos. promedio es la media ponderada de los datos proporcionados por dicha información de cada uno de los ríos.

\section{Estimación de datos}

Se realizó mediante una ecuación matemática determinística, diferencias de concentraciones de un punto a otro más cercano.

$$
\mathrm{Z}_{-} \mathrm{p}=\left(\sum_{-}(\mathrm{i}=1)^{\wedge} \mathrm{n}=\left(\mathrm{z}_{-} \mathrm{i} /\left(\mathrm{d}_{-} \mathrm{i}^{\wedge} \mathrm{p}\right)\right)\right) /\left(\sum_{-}(\mathrm{i}=1)^{\wedge} \mathrm{n} \mathrm{W}_{\text {- }}\left(\mathrm{z}_{-} \mathrm{i} /\left(\mathrm{d}_{-} \mathrm{i}^{\wedge} \mathrm{p}\right)\right)\right)
$$

La interpolación del punto problema se realiza asignando pesos a los datos del entorno en función inversa de la distancia que los separa, trata una media ponderada de los daros en función de la distancia hacía puntos más cercanos.

\section{Elección del modelo matemático unidimensional}

Son los modelos más simples y comúnmente utilizados en el análisis de la calidad de agua de ríos, estos modelos asumen que los cambios significativos que sufren los parámetros determinan la calidad del agua a lo largo del perfil longitudinal del recurso acuático (Ziemińska \& Skrzypski, 2012).

Para formular una relación determinística entre los residuos descargados al ambiente y la calidad del agua resultante, ambas en términos de un mismo compuesto, se debe considerar un volumen de río donde la coordenada x se alinea con la longitud del río, la coordenada y con su ancho, y la coordenada z con la profundidad del río.

Ciertos cuerpos de agua son considerados homogéneos considerando las variables de la calidad de agua en direcciones transversal y vertical, pues, el contaminante varía solo en la dirección del río, esta característica es conocida como unidimensional, en contraste con los cuerpos de agua en los sistemas tridimensionales. -

\section{Balance de masas}

Las fórmulas utilizadas en el balance de masas se basan en lo descrito por (Guzmán, y otros, 2011): 
La masa total del compuesto $C\left[M / L^{3}\right]$ que entra a través de la sección de control, en un intervalo de tiempo $\Delta t$ es:

$$
\text { Q.C. } \Delta t \quad \text { ec. } 1
$$

La masa total del compuesto $C\left[M /{ }_{L}^{3}\right]$ que entra a través de la sección de control, en un intervalo de tiempo $\Delta t$ es:

$$
\text { Q.C. } \Delta t \quad e c .2
$$

Donde:

Qes el caudal en el río.

De manera similar, la masa de contaminante que sale del volumen de control a través de una cara situada a una distancia $\Delta x$ de la zona de entrada está dada por:

$$
(Q+\Delta Q) \cdot\left(C+\frac{\partial C}{\partial x} \Delta x\right) \cdot \Delta x \quad e c .3
$$

A partir de las definiciones anteriores, la variación de la masa del compuesto $C$ dentro del volumen de control debido a la entrada y salida de contaminante, así como también debido a reacciones simples, sobre el intervalo de tiempo $\Delta t$ es:

$$
V . \Delta C=Q . C . \Delta t-(Q+\Delta Q) \cdot\left(C+\frac{\partial C}{\partial x} \Delta x\right) \cdot \Delta t \pm K . V . C . \Delta t \quad \text { ec. } 4
$$

Donde:

$K[1 / T]$ representa el coeficiente de una reacción de primer orden que describe la pérdida o ganancia del compuesto $C$.

Al expandir la ecuación 4 y dividir por el intervalo de tiempo, $\Delta t$, y el volumen $V(=$ A. $\Delta x)$, se tiene:

$$
\frac{\Delta C}{\Delta t}=-\frac{Q}{A} \cdot \frac{\partial C}{\partial x}-\frac{C}{A} \cdot \frac{\Delta Q}{\Delta x}-\frac{\Delta Q}{A} \cdot \frac{\partial C}{\partial x} \pm K . C \text { ec. } 5
$$

Si el límite cuando el tamaño de la separación, $\Delta \mathrm{x}$, $\mathrm{y}$ el intervalo de tiempo, $\Delta \mathrm{t}$, se aproximan a cero se define como:

$$
\frac{\partial C}{\partial t}=-\frac{Q}{A} \cdot \frac{\partial C}{\partial x}-\frac{C}{A} \cdot \frac{\partial Q}{\partial x} \pm K . C \quad e c .6
$$

O escrito en forma más reducida:

$$
\frac{\partial C}{\partial t}=-\frac{1}{A} \cdot \frac{\partial(Q \cdot C)}{\partial x} \pm K . C \quad \text { ec. } 7
$$

Las ecuaciones 6 y 7 representan la ecuación diferencial básica para el estudio de un sistema simple y no dispersivo 
Si la variable $\mathrm{C}$ representa un compuesto conservativo, $\mathrm{K}=0$. El flujo, el área perpendicular, así como el coeficiente de decaimiento pueden ser funciones de la distancia y tiempo.

Comúnmente se considera el origen del eje x en la ubicación de una descarga. De esta manera, una condición de borde característica es:

$$
C=C_{0}(t) \text { en } x=0 \text { ec. } 8
$$

Donde:

$C_{0}(t)$ es la concentración del compuesto $C$ en la localización de la descarga.

\subsection{Régimen Permanente, Compuesto No conservativo}

Las fórmulas utilizadas se basan en lo descrito por (Guzmán, y otros, 2011):

En este caso la ecuación 6 se puede escribir como:

$$
0=-\frac{Q}{A} \cdot \frac{d C}{d x}-\frac{C}{A} \cdot \frac{d Q}{d x}-K . C \quad \text { ec. } 9
$$

Si nuevamente se supone que el caudal y el área transversal son constantes, y solo se considera descargas puntuales, la ecuación 9 se puede escribir como:

$$
0=-\frac{Q}{A_{0}} \cdot \frac{d C}{d x}-K \cdot C=-u \cdot \frac{d C}{d x}-K . C \text { ec. } 10
$$

Con la condición de borde:

$$
C=C_{0} \quad \text { en } \quad x=0 \quad \text { ec. } 11
$$

La integración de la ecuación 11 permite escribir:

$$
C(x)=C_{0} \cdot \exp \left[-\frac{K}{u} \cdot x\right] \quad \text { ec. } 12
$$

Tasa de desoxigenación en función del caudal:

$$
k=1.796 Q^{-0.49} \quad \text { ec. } 13
$$

En la ecuación 12, K está en $\mathrm{d}^{\wedge}(-1)$ y el caudal Q en $\mathrm{m}^{\wedge}$ 3/s. El rango de aplicación de esta expresión es para caudales entre 0.3 y $23 \mathrm{~m}^{\wedge} 3 / \mathrm{s}$, por encima del mayor caudal, los autores de la ecuación demostraron que $\mathrm{K}$ es independiente del caudal y los valores son consistentes con las tasas encontradas por laboratorio. Por encima del rango de la ecuación 12, puede suponerse un valor constante de $0.30 \mathrm{~d}^{\wedge}(-1)$ (Autoridad Nacional De Licencias Ambientales (ANLA), 2013).

Al sustituir la expresión para la concentración C_0 se puede obtener: 


$$
C(x)=\left(\frac{Q_{R} \cdot C_{R}+Q_{D} \cdot C_{D}}{Q_{R}+Q_{D}}\right) \cdot \exp \left[-\frac{K}{u} \cdot x\right] \text { ec. } 14
$$

\section{Resultados}

Los estudios de modelación son importantes porque permiten conocer la cantidad, calidad y tipo de sustancias que ingresan en los cuerpos de agua, esto permite la fijación de condiciones particulares de descargas, así como la toma de decisiones que permitan establecer acciones para la conservación de los recursos, garantizando y promoviendo la conservación sustentable del ambiente (Montelongo et al., 2008).

De acuerdo con los datos de TPH de calidad de agua existen datos faltantes debido a que en los puntos de monitoreo del Gobierno Autónomo Descentralizado de la provincia de Orellana no se está analizando dicho factor, por lo que a continuación se describe los datos de sedimentos de cada uno de los factores que se analizaron tales como: Cadmio, Níquel, Plomo y TPH en los años establecidos.

Tabla 3 Datos de TPH en los puntos de monitoreo

\begin{tabular}{|c|c|c|c|c|c|c|}
\hline \multirow[t]{2}{*}{ ID } & \multirow[t]{2}{*}{ PUNTOS DE MONITOREO } & \multicolumn{5}{|c|}{ TPH (MG/L) } \\
\hline & & 2015 & 2016 & 2017 & 2018 & 2019 \\
\hline 1 & $\begin{array}{l}\text { Palo Azul - La Unión la } \\
\text { Moreña }\end{array}$ & 0.00 & 0.00 & 0.00 & 0.00 & 0.00 \\
\hline 2 & Sacha Norte 2 - Enokanqui & 0.00 & 0.00 & 0.00 & 0.00 & 0.00 \\
\hline 3 & Sacha Norte 1 & 33.20 & 0.00 & 0.00 & 0.038 & 0.00 \\
\hline 4 & Sacha Central & 1.40 & 0.00 & 0.00 & 15.00 & 0.00 \\
\hline 5 & Sacha 192 & 0.20 & 0.00 & 0.00 & 0.00 & 0.00 \\
\hline 6 & Nuevo Paraiso & 0.00 & 0.00 & 0.00 & 0.0078 & 0.00 \\
\hline 7 & Huashito & 0.00 & 0.00 & 0.00 & 0.0073 & 0.00 \\
\hline 8 & Estación Coca & 0.00 & 0.00 & 0.00 & 0.0095 & 0.00 \\
\hline 9 & Tigüino & 0.00 & 0.00 & 0.00 & 0.00 & 0.00 \\
\hline 10 & Puma 2 & 0.00 & 0.00 & 0.00 & 0.00 & 0.00 \\
\hline 11 & Nantu A & 0.00 & 0.00 & 0.00 & 0.0087 & 0.00 \\
\hline 12 & Pindo Central & 0.00 & 0.00 & 0.00 & 0.00 & 0.00 \\
\hline 13 & Auca Central & 0.00 & 0.00 & 0.00 & 0.00 & 0.00 \\
\hline 14 & Auca Sur & 0.00 & 0.00 & 0.00 & 0.00 & 0.00 \\
\hline 15 & Auca 51 & 0.00 & 0.00 & 0.00 & 0.00 & 0.00 \\
\hline 16 & Anaconda 1 & 0.00 & 0.00 & 0.00 & 0.0069 & 0.00 \\
\hline 17 & Yuca Central & 0.00 & 0.00 & 0.00 & 0.0028 & 0.00 \\
\hline 18 & Mini Estación Culebra & 0.00 & 0.00 & 0.00 & 0.0066 & 0.00 \\
\hline 19 & Yulebra 1 & 0.00 & 0.00 & 0.00 & 0.0076 & 0.00 \\
\hline
\end{tabular}




\section{Análisis exploratorio de datos}

Estudio de datos desde todas las perspectivas tomando en consideración las concentraciones y caudales en bruto de los ríos en los diferentes puntos y con todas las herramientas posibles, cuyo propósito es extraer cuanta información sea posible, a través de gráficos estadísticos.

Debido a que La validación de un modelo matemático implica el uso de un número de datos y pruebas con estos, misma que puede ser operacional (comparación de datos) reales con datos simulados) o conceptual. (Sámano) Los estudios de modelación son importantes porque permiten conocer la cantidad, calidad y tipo de sustancias que ingresan en los cuerpos de agua, esto permite la fijación de condiciones particulares de descargas, así como la toma de decisiones que permitan establecer acciones para la conservación de los recursos, garantizando y promoviendo la conservación sustentable del ambiente. Por lo que se analizó de manera independiente la concentración de cada contaminante.

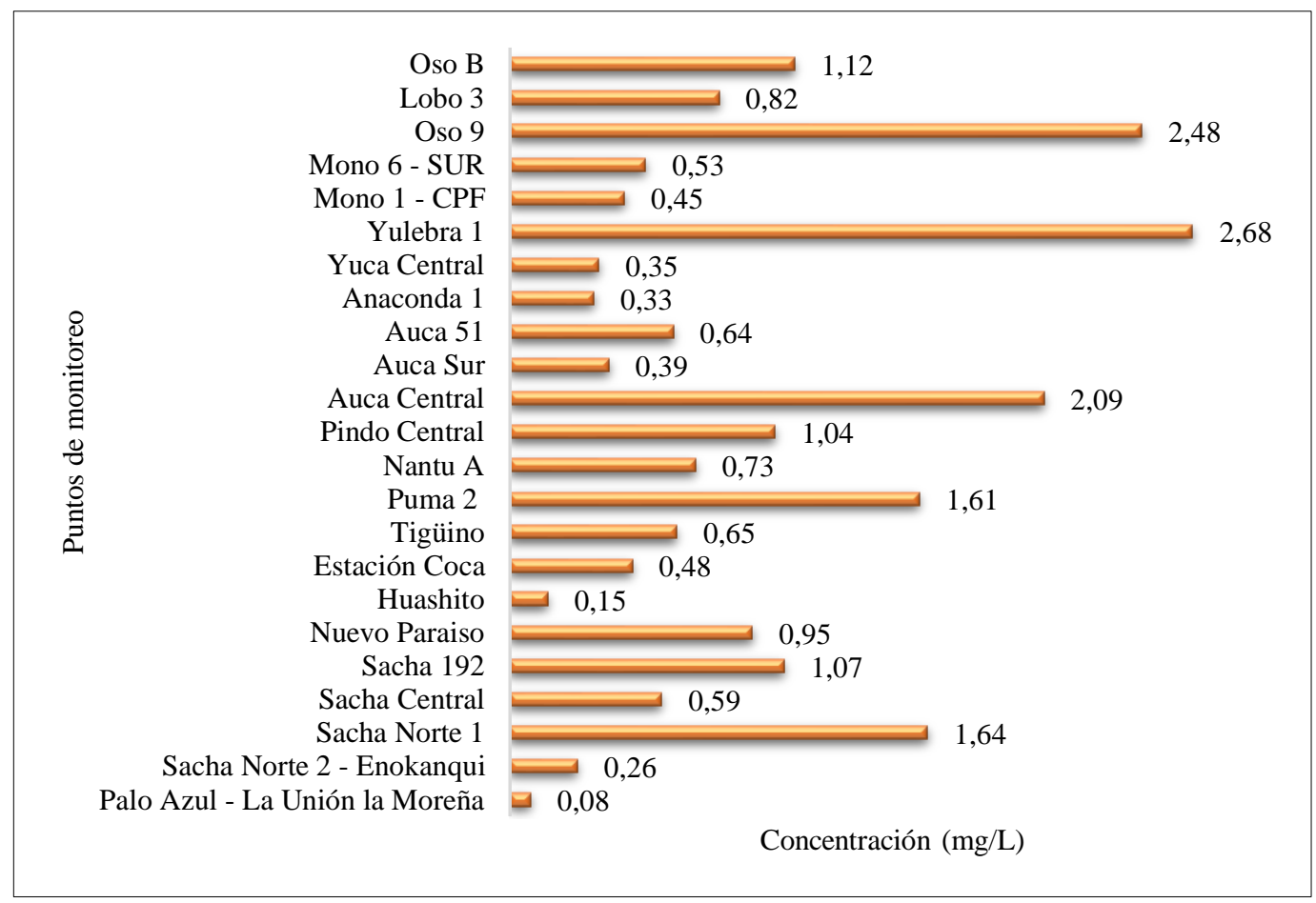

Gráfico 1. Concentración de cadmio

En el punto Yulebra 1 existió una concentración de $2.68 \mathrm{mg} / \mathrm{L}$, a través de una media ponderada, por lo que indica que hay una mayor acumulación de Cadmio, mientras que en el punto Palo Azul-La Unión la Moreña se obtuvo una concentración de $0.08 \mathrm{mg} / \mathrm{L}$, es decir una menor acumulación. 


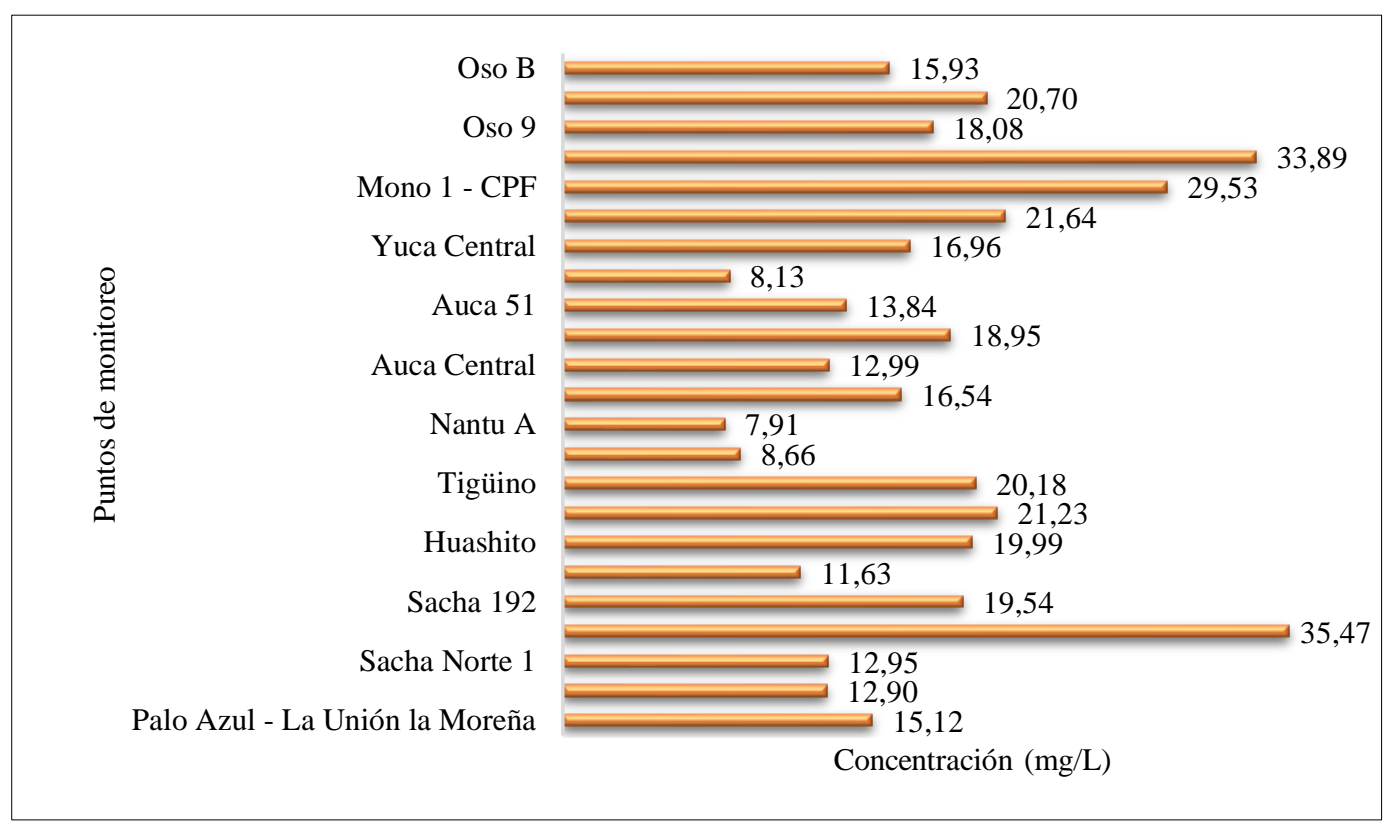

Gráfico 2. Concentración de níquel

En el punto Sacha central existió una concentración de $35.47 \mathrm{mg} / \mathrm{L}$, a través de una media ponderada, por lo que indica que hay una mayor acumulación de níquel mientras que en el punto Nantu-A se obtuvo una concentración de $7.91 \mathrm{mg} / \mathrm{L}$, es decir una menor acumulación de Níquel en el punto.

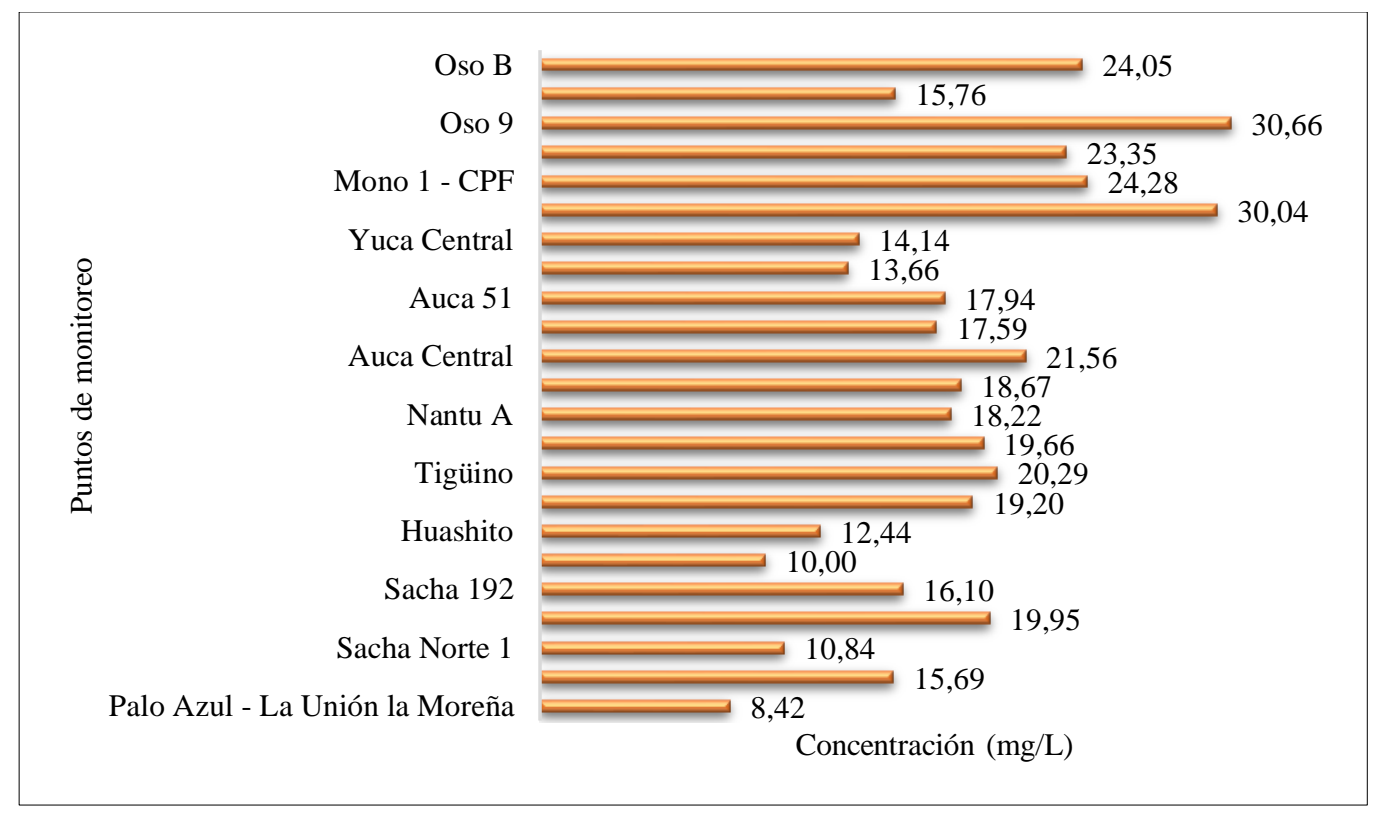

\section{Gráfico 3. Concentración de plomo}

En el punto Oso 9 existió una concentración de $30.66 \mathrm{mg} / \mathrm{L}$ por medio de una media ponderada, lo que indica una mayor acumulación de Plomo, mientras que en el punto Palo Azul-La Unión la Moreña se obtuvo una concentración de $8.42 \mathrm{mg} / \mathrm{L}$, es decir una menor acumulación de Plomo. 


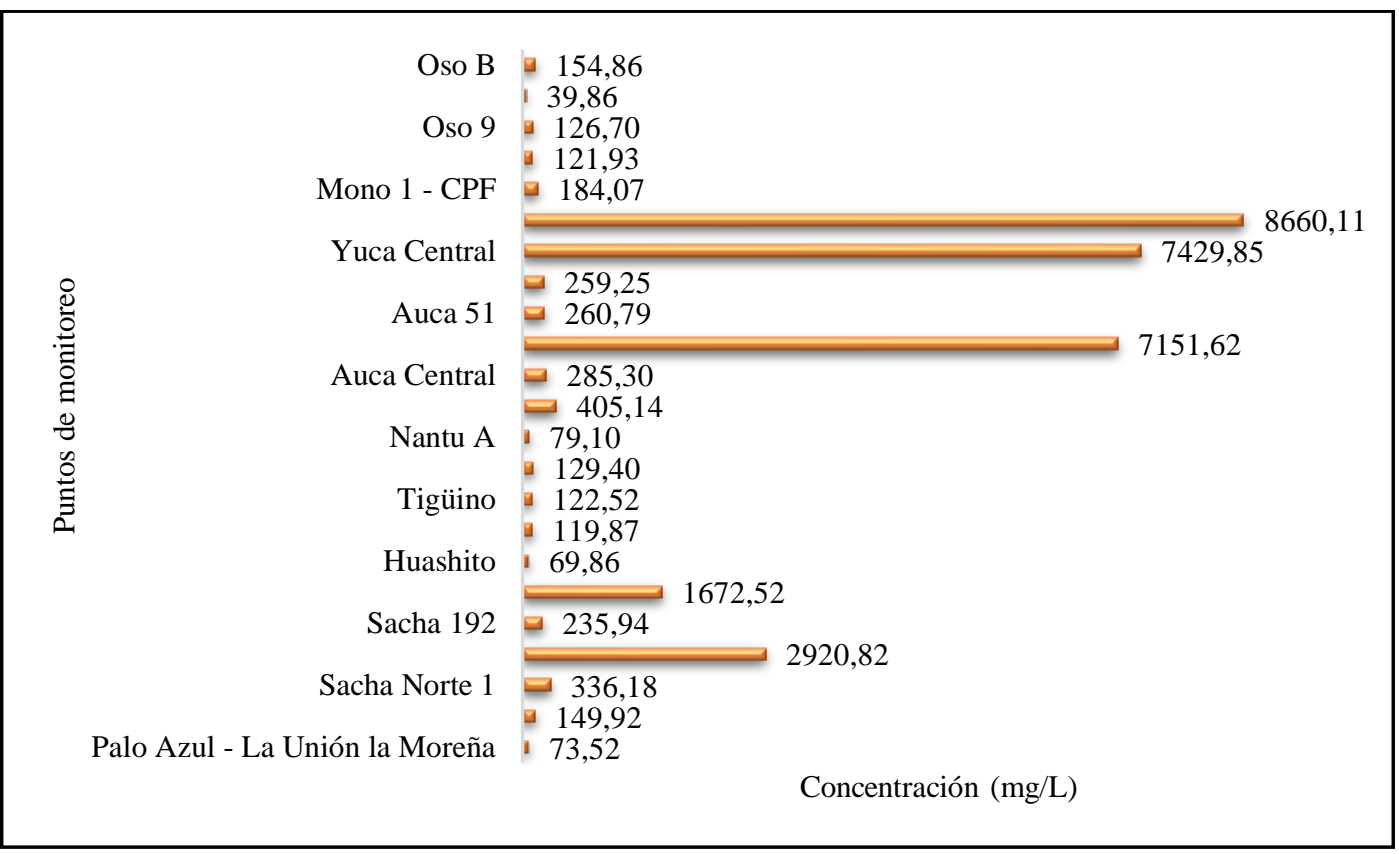

Gráfico 4. Concentración de hidrocarburos totales de petróleo

En el punto Yulebra 1 existió una concentración de $8660.11 \mathrm{mg} / \mathrm{L}$ a través de una media ponderada, por lo que indica una mayor acumulación de hidrocarburos, totales de petróleo mientras que en el punto Lobo 3 tuvo una concentración de $39.86 \mathrm{mg} / \mathrm{L}$, es decir una menor acumulación de hidrocarburos.

\section{Calidad de agua en los ríos}

La calidad de agua está definida como las condiciones fisicoquímicas de un sistema que contribuyen al mantenimiento de la biota presenta en los cuerpos de agua, garantizando el equilibrio del sistema con el ambiente (Marusic, 2013).

La presencia excesiva de contaminantes debido a las actividades extractivas, modelos de transportes, generación de energía y actividades cotidianas producen contaminantes que son transportados por las corrientes de aire y son depositados en cuerpos de agua (Owa, 2013). Índice de calidad del agua es un número que determina las características del agua por la combinación de ocho variables para dar a conocer un métodos simple y veraz (Barrabino et al., 2017).

\section{Modelo teórico general}

Por medio del uso de software especializado se identificó la dirección de los Ríos en la zona de estudio. Este es un parámetro importante para el modelo matemático La dirección se lo realiza con las herramientas de HYDROLOGY sustentado en el software (arcgis).

Se seleccionaron los ríos de primer orden, río Napo y de segundo orden río Payamino, Coca, Jivino, Huamayacu, Blanco y Sacha, debido a la cercanía de los puntos de monitoreo y a la existencia de información cartográfica de antes y después de la descarga de los contaminantes en la fuente receptora, y el caudal promedio es la media ponderada de los datos proporcionados por dicha información. 


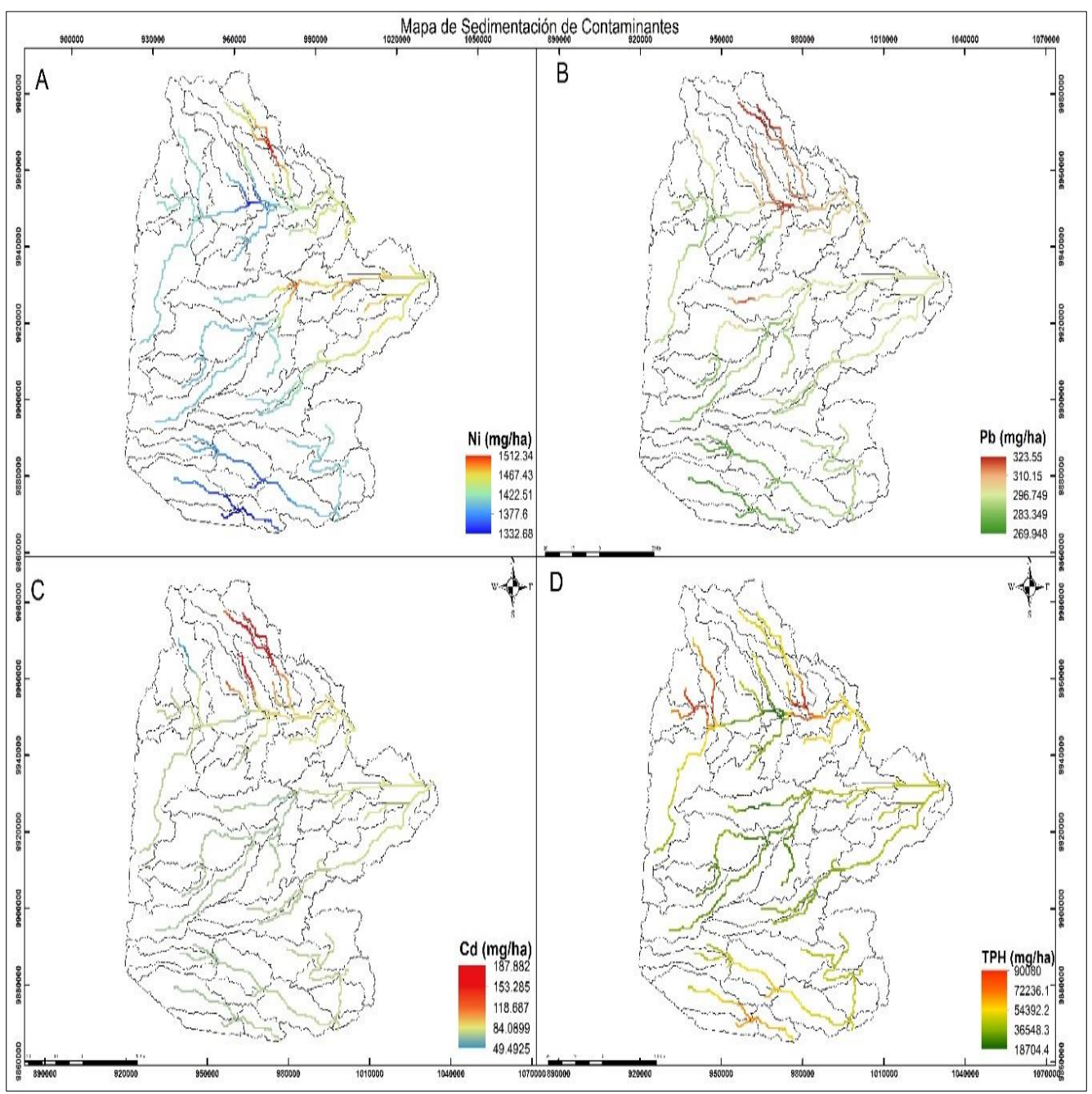

Gráfico 5. Estimación de concentración de variables

En el gráfico 5 se observa que una vez aplicado el modelo matemático en el modelamiento SWAT, el mapa A indica que la sedimentación de níquel va de 1332.68 a $1512.34 \mathrm{mg} / \mathrm{ha}$, donde las microcuencas de la zona norte y centro-este tiende a tener mayor acumulación de dicho contaminante. Por otro lado, en el mapa B, la concentración de plomo va de 269.948 a $323.55 \mathrm{mg} / \mathrm{ha}$ en las microcuencas de la zona norte. El cadmio es el contaminante que menor sedimentación presenta, de 49.49 a $187.88 \mathrm{mg} / \mathrm{ha}$ y mantienen la predominancia, al igual que en los anteriores casos, de mayor acumulación en las microcuencas de la zona norte.

En contraste a este, los hidrocarburos totales de petróleo son los que cubren un mayor rango de sedimentación de 18704.4 a 90080 mg/ha en la mayoría de las microcuencas, por lo que estos excederían la capacidad de carga, suponiendo una acumulación virtual de dichos contaminantes. Cabe señalar que se programó un período de 5 años, correspondiente al período de monitoreo, como el período de iso-balance en donde la escorrentía y precipitación garantizan una acumulación hídrica correspondiente al balance de los cuerpos hídricos subterráneos sean estos confinados o libres según la litología que los origine. 


\section{Balance de masas}

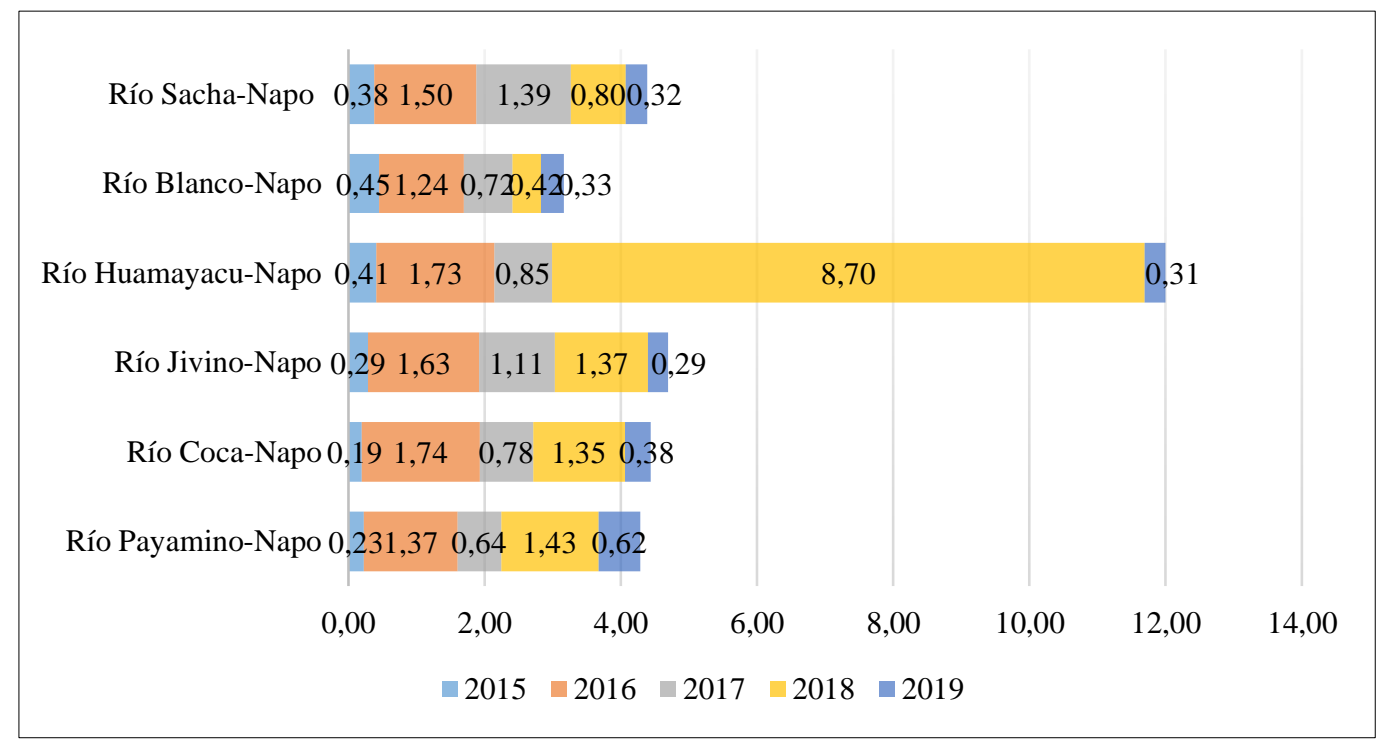

Gráfico 6. Cadmio presente en la relación del rio de primer orden con los ríos de segundo orden

Mediante el balance de masa realizado se obtiene como resultado que en la relación de contaminación del rio principal de la provincia de Orellana (río Napo) con los ríos secundarios se encuentra la mayor concentración de Cadmio en el rio Huamayacu con 8,70 mg/l ocurrido en el año 2018 el cual sobrepasa los límites permisibles, teniendo en consideración que en este año se encuentra la mayor concentración de cadmio en todos los ríos estudiados, pero existe una considerable disminución en el año 2019 con una concentración de 0,29 mg/L en el río Jivino-Napo. Lo cual se considera favorable para la población ya que el cadmio al llegar a los ríos se deposita en los sedimentos es absorbido por plantas y animales que llegando a la cadena alimentaria perjudica a los pobladores de la provincia causando daños en sus riñones, pulmones y sistema nervioso (Mero et al., 2019).

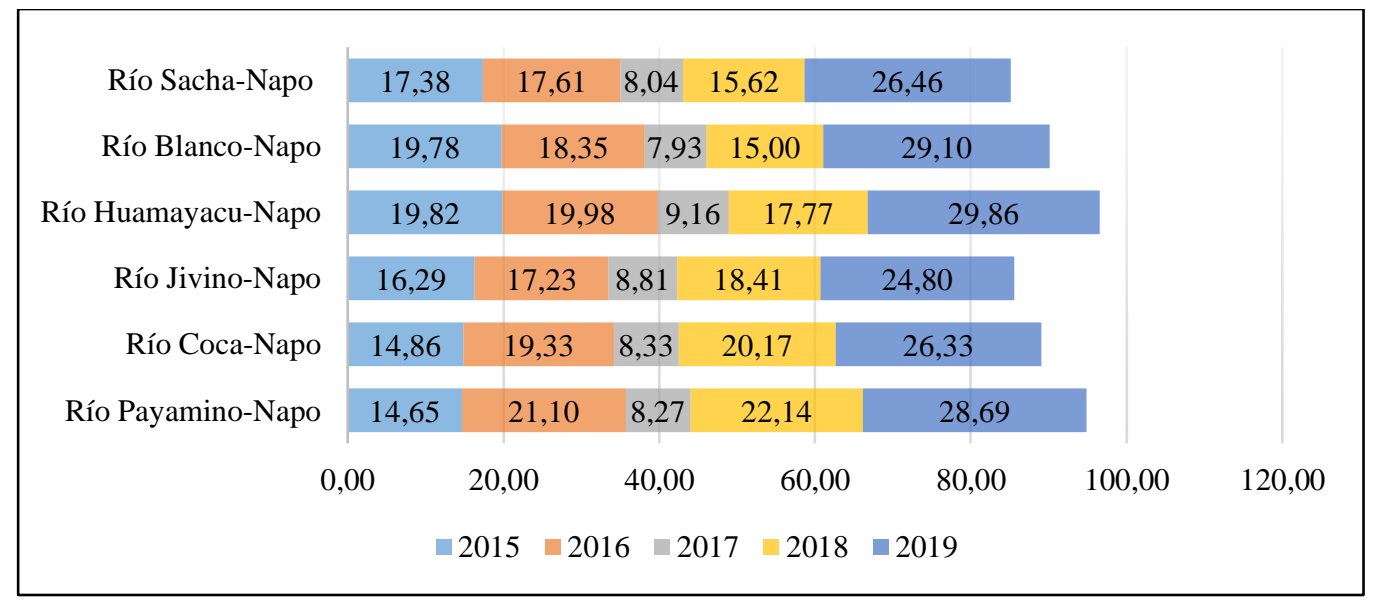

Gráfico 7. Níquel presente en la relación del rio de primer orden con los ríos de segundo orden

Él análisis en la concentración de Níquel nos indica que existe una mayor concentración la relación rio Huamayacu - Napo tiene una concentración de 29,86 mg/L siendo esta la 
mayor de todas y la menor concentración en el mismo año con 24,80 mg/L en el río Jivino-Napo.

Níquel al terminar en la superficie de agua puede disminuir el rango de crecimiento de algas ocasionando alteración en los ciclos biogeoquímicos, la retención de nutrientes, la formación y estabilidad de los sedimentos y modifican la velocidad de la corriente (Bojorge \& Cantoral, 2016).

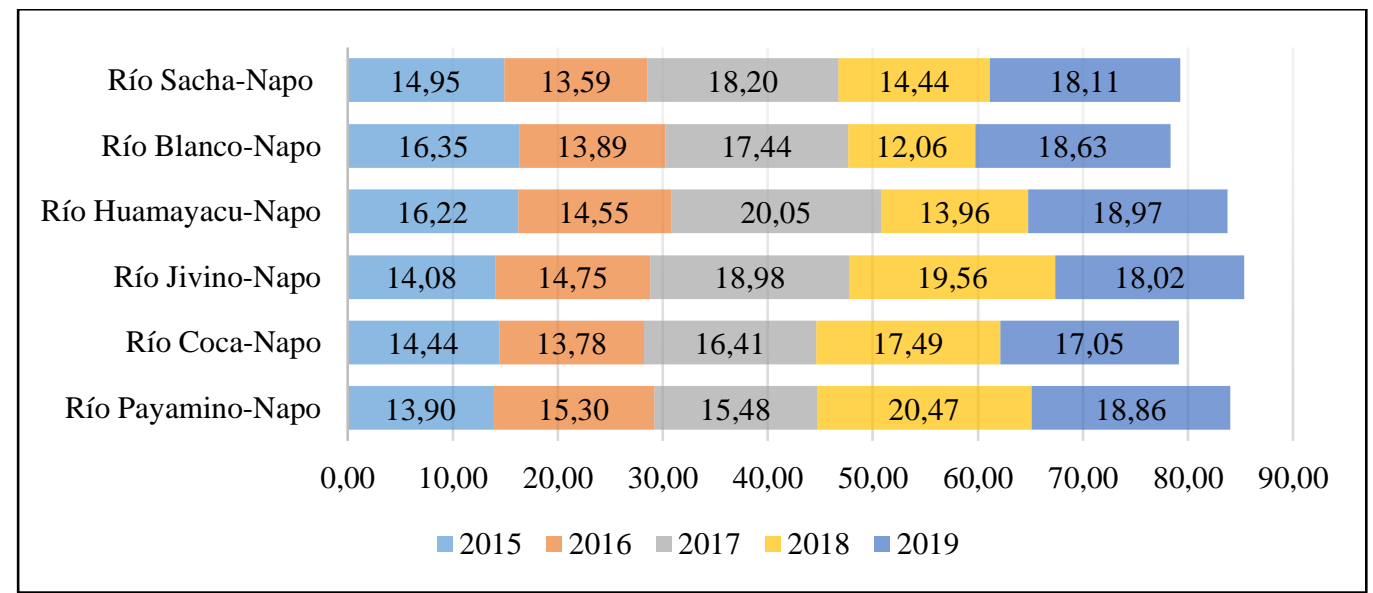

Gráfico 8. Plomo presente en la relación del rio de primer orden con los ríos de segundo orden

Con respecto a la concentración de plomo a pesar de que no exista gran variabilidad en sus concentraciones se observa que un mayor porcentaje de este metal en la relación rio Payamino-Napo en el año 2018 con 20,47 mg/L, concentración que representa un peligro ya que el plomo no es un elemento biodegradable y persiste en el agua y sedimentos acumulándose en los sistemas acuáticos ocasionando alteraciones en los organismos vivos que lo habitan con la posibilidad de que llegue a la cadena alimentaria y perjudique la salud de los pobladores.

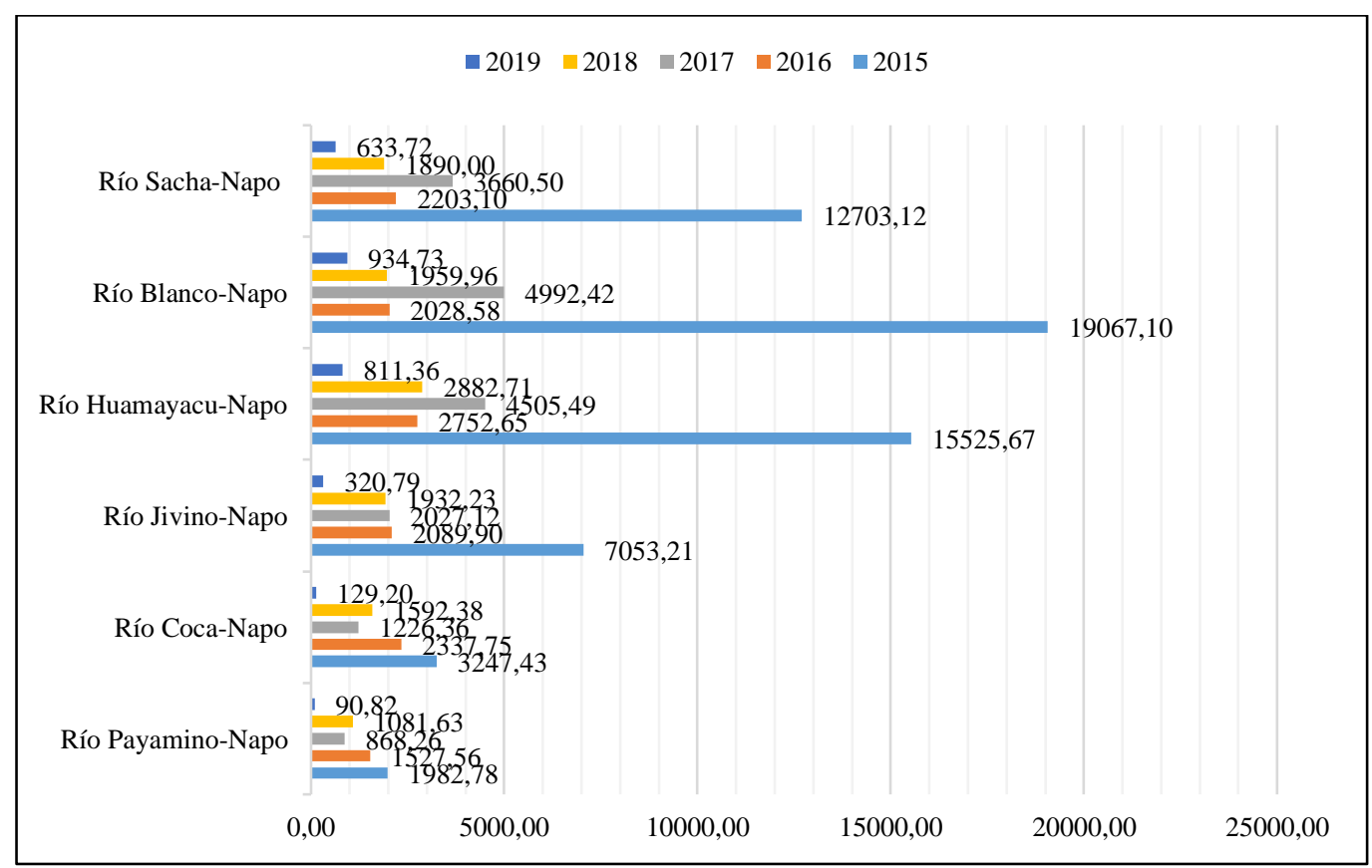

Gráfico 9. TPH presente en la relación del rio de primer orden con los ríos de segundo orden 
Mediante el diagrama se puede identificar que la mayor concentración de TPH se encuentra en la relación Río Blanco - Napo con 19057, 10 mg/L, siguiéndole la relación Río Jivino - Napo con 15525,67 mg/L y la menor concentración ocurrió en el año 2019 relación rio Coca-Napo por lo que se puede evidenciar una descendiente actividad petrolera en la localidad o a la vez un mejor tratamiento.

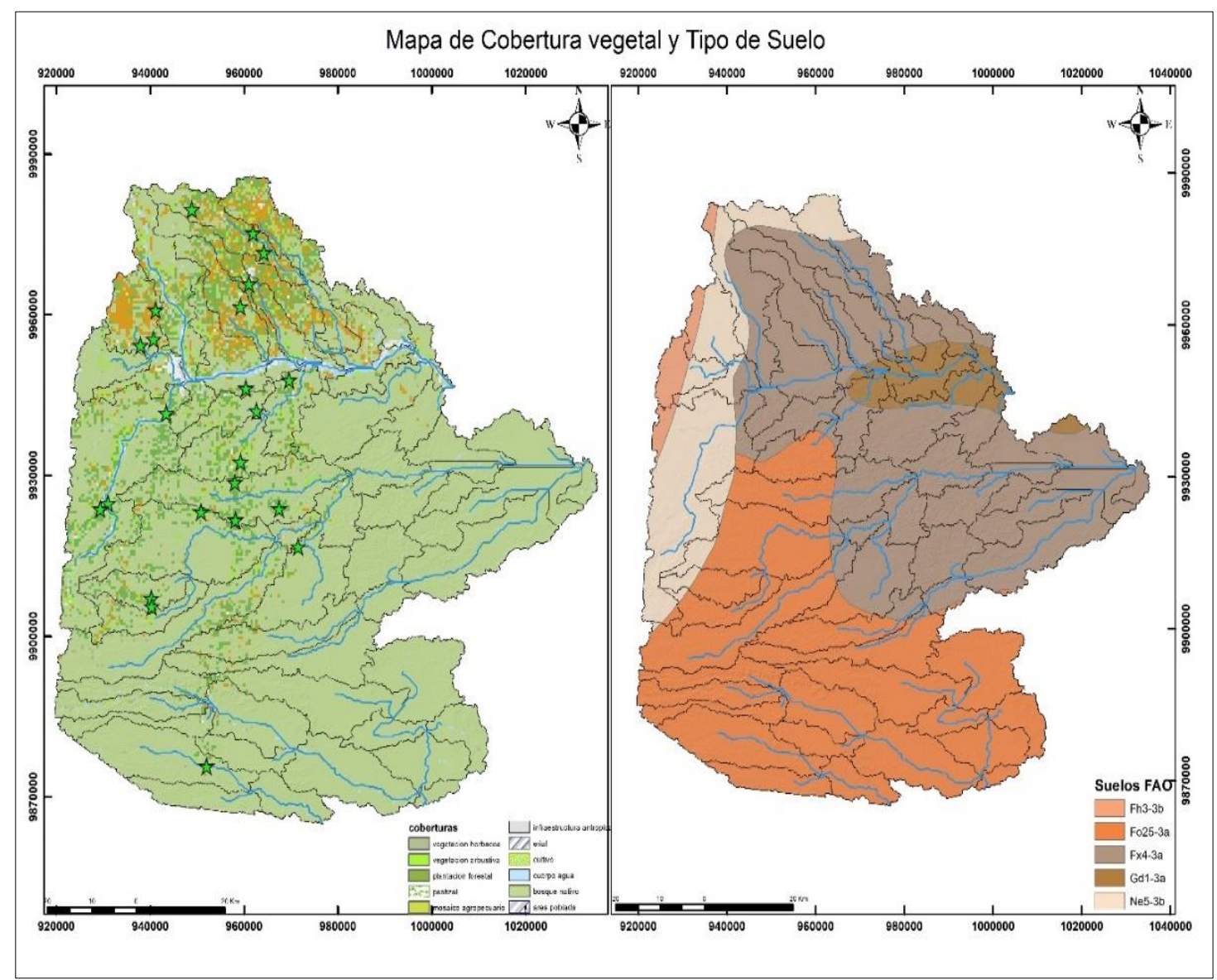

Gráfico 10. Mapa de cobertura vegetal y tipo de suelo

En el gráfico 10 se observa que el mapa A las coberturas vegetales con mayor porcentaje dentro del área de estudio son los de tipo bosque nativo, seguido de plantaciones forestales; adicional a esto se observa que la vegetación agropecuaria y cultivos en general son los que aprovechan las áreas colindantes a los cuerpos hídricos y mismas en que se desarrollan determinados asentamientos humanos en la zona norte.

Por otro lado, el mapa B indica el tipo de suelo predominante Fo25- $3^{\text {a }}$ asociado a suelos Acrisoles pinílicos y oórticos, y Fx4-3 a asociados a Glendisoles distríticos y pliníticos según la clasificación correspondiente de la Organización de Naciones Unidas para la Agricultura y la alimentación que corresponden a los órdenes taxonómicos de inceptisoles, ultisoles, entisoles y andosoles correspondientemente. 


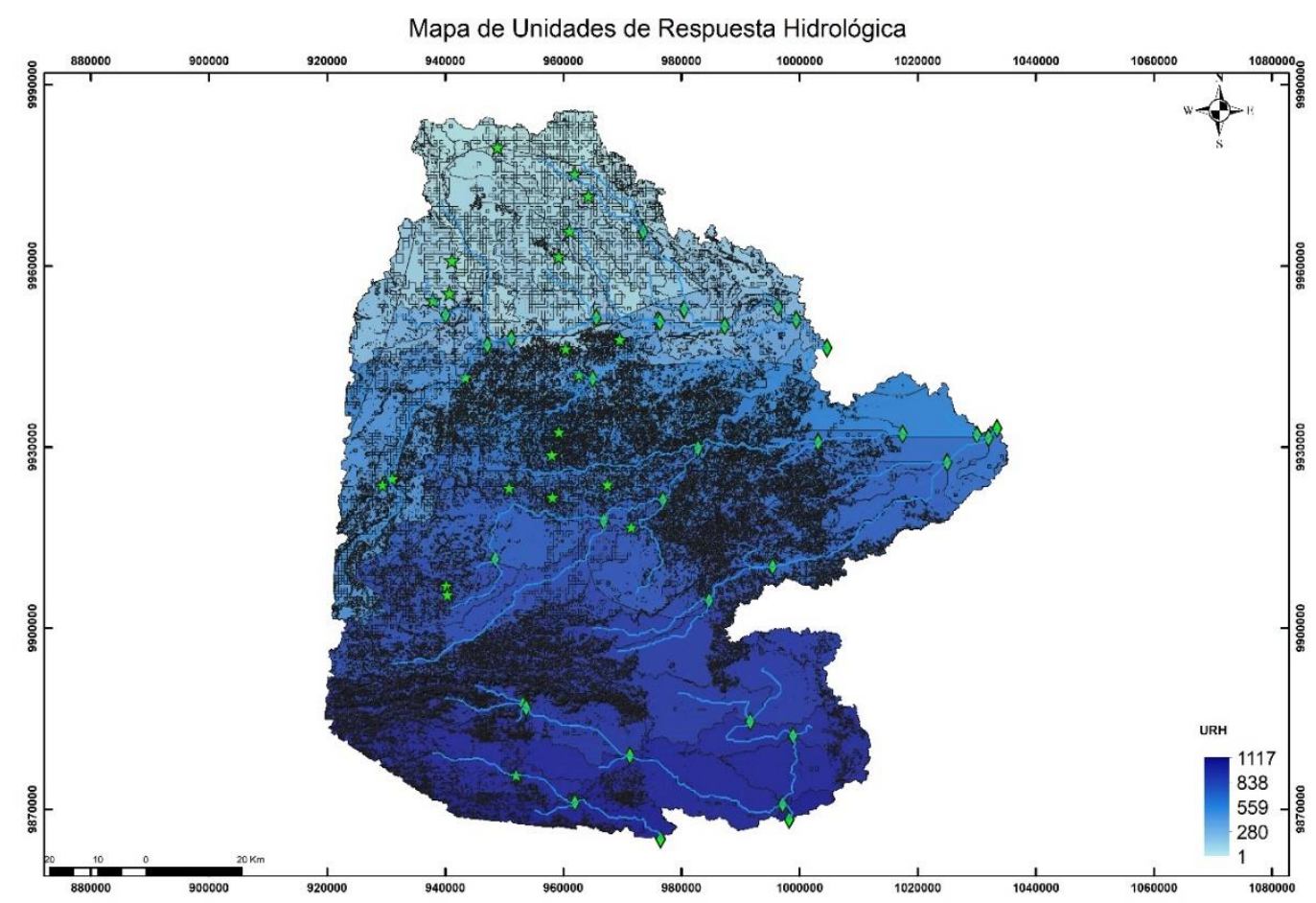

Gráfico 11. Mapa de unidades de respuesta hidrológica

Las unidades de respuesta hidrológica (URH) nos indican homogeneidad en términos de respuesta hidrológica y características geo-climáticas: la pendiente (la cual determina la velocidad de la escorrentía), la elevación (de esta depende la precipitación) y las condiciones meteorológicas (temperatura, precipitación, velocidad, dirección del viento, evo transpiración y radiación solar), Estas permiten determinar las propiedades dominantes y como indica el gráfico 11 el mapa posee una mayor generación de escorrentía y acumulación de agua en la zona baja de la subcuenca es decir en la forma semi - distribuida acorde a los caudales, tipo de suelo y pendiente que son los factores principales a los que se le atribuye en el modelo. Esto va ligado con la cantidad de sedimentación promedio anual por hectárea ya que es inversamente proporcional a las condiciones del ciclo hidrológico obtenido por el modelo.

\section{Conclusiones.}

- Este estudio evidencia la contaminación por metales pesados presente durante el periodo 2015-2019 ocasionado por actividades petroleras realizadas en la provincia, además, mediante la aplicación del modelo matemático se realizó una proyección de la concentración de los diferentes metales pesados en las subcuencas de la provincia.

- Se realizó un análisis exploratorio de datos para la aplicación del modelo matemático de diagnóstico de calidad de agua y sedimentos, donde se identificó mediante la aplicación de gráficos estadísticos y una media ponderada la mayor concentración de los diferentes puntos de monitoreos analizados, en donde cadmio se encontró en mayor concentración en el punto Yulebra 1 con 2,68 mg /L, níquel tuvo su mayor concentración en el punto Sacha central con 35,47 mg 
/L, plomo su mayor concentración fue en el punto Oso 9 con 30,66 mg / L y finalmente la mayor concentración de hidrocarburos totales de petróleo se encontró en el punto Yulebra 1 con 8660,11 mg /L.

- Se estableció un modelo hidrológico mediante el cual se identificó la dirección de los ríos de primer orden rio Napo y de segundo orden río Payamino, río Coca, río Jivino, río Huamayacu, río Blanco y río Sacha obteniendo así una media ponderada la cual vendría a ser el caudal promedio de los ríos antes y después de la descarga siendo el rio rio Napo el de mayor caudal con 42,50 『(m》^3/s) seguido del rio de segundo orden el rio Payamino con 40,34 $\left(\mathrm{m}^{\wedge} 3 / \mathrm{s}\right)$ y aplicando una ecuación determinística se realizó la diferencia de concentraciones de un punto a otro más cercano.

- Se evaluó la calidad de los ríos de primer y segundo orden mediante la aplicación del modelo matemático, en donde nos indica que la sedimentación de Ni que va de 1332.68 a $1512.34 \mathrm{mg} / \mathrm{ha}$, tiene a acumularse en la zona norte centro, la concentración de $\mathrm{Pb}$ que va de 269.948 a $323.55 \mathrm{mg} / \mathrm{ha}$ se acumula en la zona norte, el Cd es el contaminante que menor sedimentación presenta, de 49.49 a $187.88 \mathrm{mg} / \mathrm{ha}$ tiende a acumularse en la zona norte y los TPH es el contaminante con mayor grado de sedimentación de 18704.4 a $90080 \mathrm{mg} / \mathrm{ha}$ en todas las zonas, estableciendo así que los datos obtenidos exceden la capacidad de carga, suponiendo así una acumulación virtual de dichos contaminantes analizados en los ríos.

\section{Referencias bibliográficas}

Arreguín, F., Alcocer, V., \& Hernández, D. (2010). Model ación de redes de agua potable con enfoques determinísticos y estocásticos. Tecnología y Ciencias del Agua, antes Ingeniería hidráulica en México, 1(4), 119-136.

Autoridad Nacional De Licencias Ambientales (ANLA). (2013). Metodología para la definición de la longitud de influencia de vertimientos sobre corrientes de agua superficial. Bogotá D.C.: Ministerio de Ambiente y Desarrollo Sostenible. Obtenido de https://www.anla.gov.co/documentos/ciudadania/03_partic_ciudadana/conpub/Metodologia_-_Longitud_de_Influencia_de_Vertimientos.pdf

Barrabino, A., Keleşoğlu, S., Eftekhardadkhah, M., Simon, S., \& Sjöblom, J. (2017). Enhanced Sedimentation and Coalescence of Petroleum Crude Oil Emulsions by New Generation of Environmentally Friendly Yellow Chemicals. Journal of Dispersion Science and Technology, 38(12), 1677-1686. doi:https://doi.org/10.1080/01932691.2015.1004410

Bojorge, M., \& Cantoral, E. (2016). La importancia ecológica de las algas en los ríos. Hidrobiológica, 26(1), 1-8.

Gobierno Autónomo Descentralizado de la Provincia de Orellana. (2019). Plan de Desarrollo y Ordenamiento Territorial. Gobierno Autónomo Descentralizado de 
la Provincia de Orellana. https://www.gporellana.gob.ec/wpcontent/uploads/2017/03/Plan_de_ordenamiento_2016.pdf

Guzmán, G., Thalasso, F., Ramírez, E., Rodríguez, S., Guerrero, A., y Avelar, F. (2011). Evaluación espacio-temporal de la calidad del agua del río San Pedro en el Estado de Aguascalientes, México. Revista internacional de contaminación ambiental, $27(2), 89-102$.

Marusic, G. (2013). A study on the mathematical modeling of water quality in "rivertype" aquatic systems. Wseas transactions on fluid mechanic, 8(2), 80-89. doi:10.13140/RG.2.1.4334.5125

Mero, M., Pernía, B., Ramírez, N., Bravo, K., Ramírez, L., Larreta, E., \& Egas, F. (2019). Concentración de Cadmio En Agua, Sedimentos, Eichhornia crassipes Y Pomacea canaliculata en el Río Guayas (Ecuador) y sus Afluentes. Revista Internacional de Contaminación Ambiental, 35(3), 623-640. doi:10.20937/RICA.2019.35.03.09

Montelongo, R., Gordillo, A., Otazo, E., Villagómez, J., Acevedo, O., \& Prieto, F. (2008). Modelacion de la calidad del agua del río Tula, Estado De Hidalgo, México. Dyna, 75(154), 5-18.

Osina, M. (2011). Evaluación de la Calidad de las Aguas del Río KATARI, La Paz, Bolivia, mediante un modelo matemático. [Tesis de grado, Universidad Mayor de San

Andrés] https://books.google.com.ec/books?id=1P5sBgAAQBAJ\&printsec=frontcover\& redir_esc $=\mathrm{y} \# \mathrm{v}=$ onepage $\& \mathrm{q} \& \mathrm{f}=$ false

Owa, F. (2013). Water Pollution: Sources, Effects, Control and Management. Mediterranean Journal of Social Sciences, 4(8), 65-68. doi:10.5901 / mjss.2013.v4n8p65

Ziemińska, A., \& Skrzypski, J. (2012). Review of mathematical model of water quality. Ecology Chemistry Engineer, 19(2), 197-211. doi:10.2478/v10216-011-0015-x

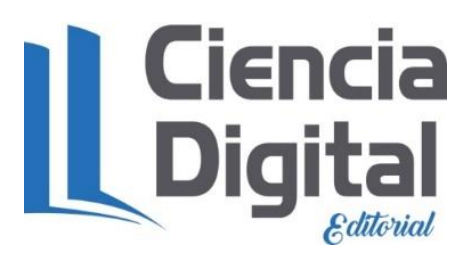




\section{PARA CITAR EL ARTÍCULO INDEXADO.}

Rosero Erazo, C. R., Yaulema Garces, F. M., \& Yaulema Castañeda, J. L. (2021). Modelo matemático estocástico para diagnosticar calidad de agua y sedimentos en zonas influenciadas por la actividad petrolera en la provincia de Orellana. ConcienciaDigital, 4(3), 177-195. https://doi.org/10.33262/concienciadigital.v4i3.1789

\section{Ciencia}

El artículo que se publica es de exclusiva responsabilidad de los autores y no necesariamente reflejan el pensamiento de la Revista Conciencia Digital.

El artículo queda en propiedad de la revista y, por tanto, su publicación parcial y/o total en otro medio tiene que ser autorizado por el director de la Revista Conciencia Digital.

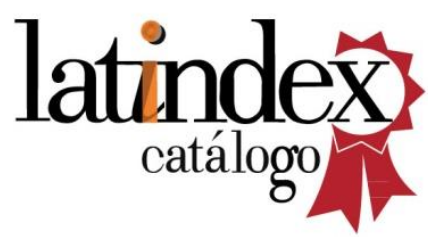

